\title{
Development of Support Vector Machine Model to Predict Stability of Slopes Based on Bound Theorems
}

\author{
D.Ramya $^{\# 1}$, S.Vinodhkumar ${ }^{* 2}$,

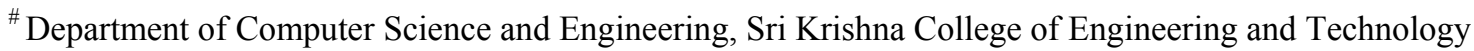 \\ Coimbatore, India \\ ${ }^{1}$ ramya2d@gmail.com \\ * Department of Civil Engineering, Kongu Engineering College \\ Perundurai, India \\ ${ }^{2}$ shansvino@gmail.com
}

\begin{abstract}
Slopes on the earth are either man made or outcomes of geological and geomorphological phylogeny. The determination of stability of slope is a complex process in geotechnical engineering. Statistical models or empirical models can be employed effectively when underlying phenomena are not well defined. In this study, the support vector machine (SVM), a kind of soft computing modeling technique, has been used to predict minimum factor of safety based on upper and lower bound theorems. The effects of various learning functions are examined. The study confirmed that SVM can be employed for prediction of stability of slopes.
\end{abstract}

Keyword- Bound theorems, Support Vector Machines, Stability of Slopes, Learning functions, soft computing modelling technique, Limit Analysis

\section{INTRODUCTION}

Stability of slope is core part in designing earth dams, carrying out excavation, making landfills and moving earth. The stability of slopes of clayey soil depends on soil shear strength parameters, unit weight of the soil, geometry of slope and pore-water pressure in the soil. Basically, there are two parameters that are important in slope analyses. They are factors that increase shear stress and factors that reduce the strength of soil. The stability of slope is usually measured with Factor of Safety i.e. ratio of shear strength of soil to the mobilized shear strength. There are two kinds of slopes, finite slope and infinite slope. Finite slope has definite boundary conditions while infinite slopes are of unlimited extended [1].

Limit equilibrium methods are widely used to solve stability problems associated with the slopes. Friction circle method assumes an arc shaped failure surface along which soil mass is rotated about the friction circle. By drawing a force triangle for cohesion, unit weight of soil and reaction force, mobilized cohesion along the failure surface is found out [1]. Slip circle method considers the failure surface as a circular curve and entire soil mass is divided into number of strips. Moments are calculated in two cases namely actuating moment and restoring moment by considering cohesion, weight of rotating soil mass and reaction force. Factor of safety is found out by dividing restoring moment by actuating moment. The critical failure surface is a surface that has very minimum factor of safety that can be achieved by trial and error method [6]. Bishop developed a method in which forces acting on sides of each slices are included in analysis thereby satisfying the requirements of global moment of equilibrium and moment of equilibrium of each slice [3]. In 1967, Spencer modified the Bishop's method by considering the forces acting on either side of the slope are parallel to each other and satisfies the force and moment equilibrium conditions [4]. Bell established a method that could satisfy all equilibrium conditions. The rotating surface may be of any shape and rotating soil mass is divided into number of slices. By drawing free body diagram factor of safety is estimated [5]. Stability Charts are obtained from above said methodologies of slope analyses which consist of set of curves. The curves are employed to find the factor of safety [1]. Complex soil profiles, phreatic surfaces can easily be incorporated in these analyses. However, the main drawback of these analyses is assumptions involved in the derivation of equation for Factor of Safety. Sometimes slopes that are having factor of safety higher than one are failed. This necessitates evaluation of a new method of analysis called limit analysis which is superior to limit equilibrium analysis in multiple aspects.

Limit analysis considers soil as perfectly plastic material. Based on this assumption two theorems (upper bound and lower bound) are developed. Upper bound theorem states that the set of external loads act on a failure criterion and the work done by the external loads in an increment of displacement equals the work done by the internal stress, the external loads obtained are not lower than the true collapse load. The lower bound theorem can be defined as if an equilibrium distribution of stress covering the whole body can be found that 
balances a set of external loads on the stress boundary and is nowhere above the failure criterion of the material, the external loads are not higher than true collapse loads. Upper bound theorem can be obtained by ignoring the equilibrium condition whereas lower bound theorem can be obtained by ignoring compatibility condition [2].

\section{LITERATURE REVIEW}

In recent years, soft computing modelling techniques are being extensively used in geotechnical engineering [13]. Among various soft computing techniques Artificial Neural Networks (ANN) and Support Vector Machines (SVM) are generally used.

Pal, Mahesh, and Surinder Deswal modelled the load carrying capacity of pile foundation using SVM and ANN. They developed SVM model based on radial basis function and polynomial kernel using 81 data sets for training and 24 data sets for testing. The performance of SVM and ANN were compared and SVM models functions better than ANN models [9].

Kuo, Y. L., M. B. Jaksa, A. V. Lyamin, and W. S. Kaggwa developed an ANN model to predict Bearing Capacity of strip footing in layered soil. In this analysis upper bound theorem and lower bound theorem were used to find the bearing capacity of footing and result of which are used to develop multi-layer perception ANN. This ANN model is compared with Multiple Regression Analysis (MRA) model and found that ANN models performed better than MRA model [8].

Prediction of settlement of foundations using SVM was investigated by Samui, Pijush [12]. SVM were used as a regression tool to find settlement using various kernel functions such as radial bias function, polynomial function and spline function. Sensitivity analysis was attempted to know the importance of each factor that induces settlement and found that Standard Penetration Test resistance was the prime factor.

Goh, Anthony TC, and S. H. Goh used SVM as classification tool for liquefaction Analyses [7]. They used Cone Penetration Test data along with soil and seismic characteristics to check the possibility of liquefaction to happen and achieved a classification success rate of $98 \%$.

Tinoco, Joaquim, A. Gomes Correia, and Paulo Cortez have used SVM to predict uniaxial compressive strength of jet grouting columns using 472 data sets and obtained $\mathrm{R}^{2}$ value of 0.51 which was due to the fact the large amount of non-linearity exists among the variables [15].

Vinodhkumar S and Balaji S developed an ANN model to predict California Bearing Ratio (CBR) of Black Cotton Soil Subgrade Stabilized with Flyash and Geotextile. In this research an attempt as mode to develop optimized ANN model by altering various learning algorithms and number of neurons in hidden layer. It was concluded that Levenberg-Marquardt back propagation model possessed $\mathrm{R}$ value 0.98695 which was the highest value of $\mathrm{R}$ among the several other algorithms [17].

Cho, Sung Eun using ANN, developed a probabilistic model of stability analyses of slopes by strength reduction method. Latin hypercube sampling technique was used to produce the soil properties and Monte Carlo simulation coupled with ANN was incorporated to develop the model [4].

Samui, P., and D. P. Kothari utilized least square support vector machine (LSSVM) as regression tool and classification tool for slope stability analysis and compared it with ANN model. The developed model was superior to the normal SVM models in terms of its performance [10].

Samui, Pijush developed a SVM model for slope stability analysis using different kernel functions such as polynomial functions of degree two, radial bias function and spline function. The effectiveness of model was calculated from root-mean square-error (RMSE) and mean-absolute-error (MAE) [11].

Zhao, Hong-bo developed a SVM-based reliability analysis of slope using first-order second-moment method (FOSM) and demonstrated through homogenous and non-homogenous slopes. It was concluded that The SVMbased FOSM performed well in stability analysis of slopes [18].

However, some major shortcomings of ANN include slow convergence speed, poorer generalizing performance, achieving local minimum and over-fitting issues [11].

Due to above stated reasons, in this study SVM are employed to predict factor of safety of slopes. Slope analyses are done using bound theorems.

The support vector machine (SVM) is evolved from the statistical learning theory proposed by Vapnik which is an effective tool that can be used to develop generalization performance due to the fact that it assumes a structural risk minimization (SRM) induction principle that aims at minimizing a bound on the generalization error of a model rather than minimizing the error on the training data only [16].

The research has the following aims:

1. To examine the possibility of the SVM model for predicting the stability of slopes.

2. To rank the factors that affects the stability of slopes by carry out a sensitive analysis.

3. To implement SVM model based on bound theorems.

4. To evaluate various kernel functions. 


\section{III.SUPPORT VECTOR MACHINES AS REgRESSION TOOL}

SVM is generally used as a classification tool but it can also act as regression tool when a loss function $\varepsilon$ is incorporated. While using SVM as a regression tool, the following possible ways shall be considered. Using linear functions that are well defined in high dimensional space, regression shall be attained. Vapnik's loss function is used to estimate the structural risk minimization thereby solving the regression. SVM achieves generalization by reducing the error in training set [10].

In this research, a nonlinear function is assumed as the critical slope failure surface can be a nonlinear curve. This has to be mentioned here that the previous SVM models developed are based on linear functions. The incorporation of nonlinear function ensures that this slope stability model can perform better than the SVM models based on linear functions.

Let the function be,

Where,

$$
f(x)=(w \cdot x)^{2}+b
$$

$$
\begin{aligned}
& f(x)=\text { Factor of safety of slope }(\text { FoS }) \\
& w=\text { a vector contains adjustable weight } \\
& b=\text { residual errors } \\
& x=\text { factors influences the stability of slopes }
\end{aligned}
$$

In order to avoid explicit formation of nonlinear regression, kernel functions are needed to include in the algorithm.

The kernel functions that are commonly found in the literatures are homogeneous and non-homogeneous polynomial, radial basis function. In this research, in addition the above mentioned kernels, Gaussian function, and sigmoid function are also included.

\section{IV.METHODOLOGY}

The main target of this research work is to device the above methodology in the problem of slope analysis. The data set consists of 630 different input and output statements are generated using permutations and combinations which are practically viable. The inputs and corresponding output are computed using MSEXCEL. The input parameters that have been selected are highly related to the geotechnical parameters and the geometry of slope. The input parameters are cohesion $(C)$, Angle of internal friction $(\phi)$, Height of Slope $(H)$, Unit weight of slope $(\gamma)$, Slope angle $(\beta)$. Generally increase in cohesion and angle of internal friction improve the stability of slope while the increase in slope angle and height of slopes tend to decrease the stability of slopes. The output from the model is Factor of Safety (F) which is computed based on the bound theorems.

The slope angle range from 4:1 to $1.5: 1$, the range of slope height is between $3 \mathrm{~m}$ to $10 \mathrm{~m}$, the angle of internal friction is between $0^{\circ}$ to $45^{\circ}$, the cohesion is between 0 and $100 \mathrm{kN} / \mathrm{m}^{2}$ and the soil unit weight is between 12 and $24 \mathrm{kN} / \mathrm{m}^{3}$. Fixing the penalty weight (C) and $E$ is a herculean process which is accomplished by trial and error method. The penalty weight is increased from 20 to 60 and for each penalty weight $\varepsilon$ is assigned from 0.01 to 0.05 . These ranges are arbitrarily fixed to systemize the trial and error process. A sensitivity analysis has been exercised to extract the cause and effect relationship between the inputs and outputs of the SVM model. Sensitivity is the average of ratio of percentage change in output to the percentage change in input [12]. The sensitivity analysis has been done on the best model developed using the above mentioned techniques by varying each and every input parameters to $10 \%$ and sensitivity is measured by the end of each variation in input parameter. The entire regression analysis and sensitivity analysis are done with the help of MATLAB of version R2011a.

\section{PERFORMANCE ANALYSIS OF SVM MODEL}

The performance of the SVM models constructed in this work are ranked by measuring coefficient of correlation (R), the root-mean-square error (RMSE) and the mean-absolute-error (MAE). Coefficient of correlation is the ratio of number of predicted data that equals the actual data to the total number of data. This is a measure of accuracy of prediction. A higher R value indicates the better accuracy of model. R-square is the ratio of the sum of squares of the regression and the total sum of squares. It indicates the accumulation data in the line of fit. MAE is the average of modulus of errors. A lower value of RMSE and MAE is always preferable as it indicates the predicated data is closer to the line of fit.

Various combinations of $C$ and $\varepsilon$ are used to obtain the best performing SVM models which are shown in table 1. The sigmoid function achieves higher $\mathrm{R}$ value in training although it is the second highest $\mathrm{R}$ value in training. Figs 1 to 5 depict the predicted and measured values of each kernel function during testing and training.

Fig 6 shows the RMSE and MAE values of best performing models obtained from each kernel function. It can be observed from the fig 6 that sigmoid function produces lower value of RMSE and MAE compared to the other models and hence it is chosen for doing sensitivity analysis. Fig 7 shows the results of sensitivity analysis and it 
can be inferred that the hierarchy of factors affecting slopes is height of slope $(H)$, slope angle $(\beta)$, cohesion $(C)$ and angle of internal friction $(\phi)$, and unit weight $(\gamma)$.

TABLE I

Performances of Kernel Functions

\begin{tabular}{|c|c|c|c|c|c|}
\hline $\begin{array}{c}\text { Kernel } \\
\text { Function }\end{array}$ & $\mathbf{C}$ & $\varepsilon$ & $\begin{array}{c}\text { Training } \\
\text { Performance }\end{array}$ & $\begin{array}{c}\text { Testing } \\
\text { Performance }\end{array}$ & $\begin{array}{c}\text { No. of } \\
\text { Support } \\
\text { Vectors }\end{array}$ \\
\hline $\begin{array}{c}\text { Non homogeneous } \\
\text { polynomial of order 2 }\end{array}$ & 20 & 0.02 & 0.96192 & 0.93993 & 81 \\
\hline $\begin{array}{c}\text { homogeneous } \\
\text { polynomial of order 2 }\end{array}$ & 32 & 0.03 & 0.93171 & 0.93544 & 45 \\
\hline Radial basis function & 40 & 0.03 & 0.96965 & 0.94455 & 32 \\
\hline Gaussian function & 29 & 0.04 & 0.96101 & 0.95125 & 48 \\
\hline sigmoid function & 54 & 0.05 & 0.96773 & 0.94862 & 78 \\
\hline
\end{tabular}

Training: $\mathrm{R}=0.96192$

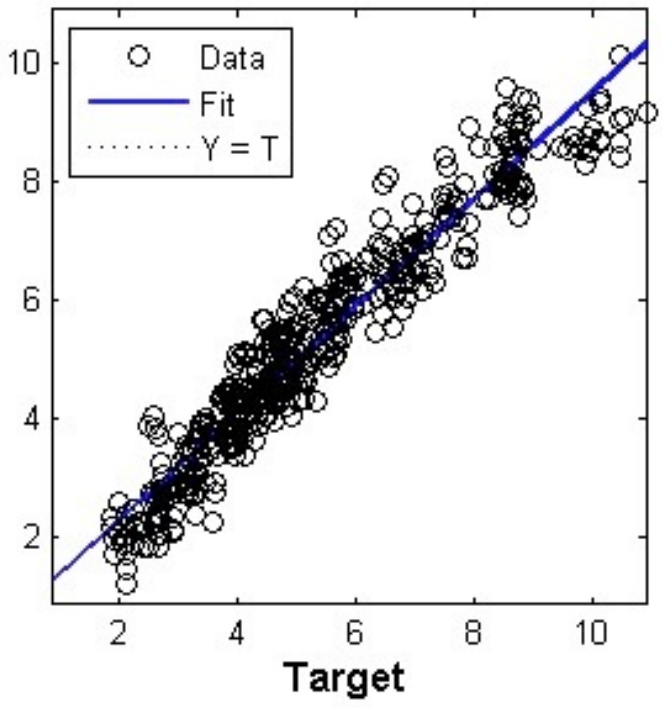

Test: $\mathbf{R}=0.93993$

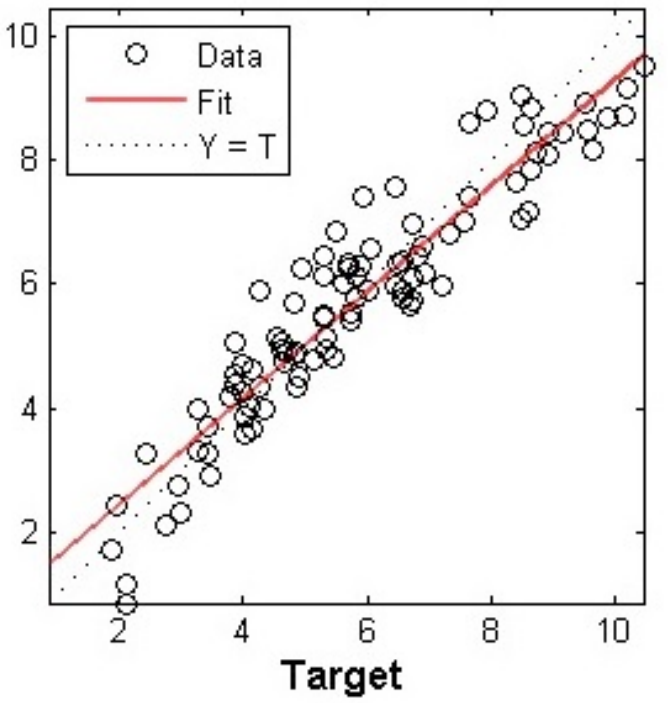

Fig. 1. Non homogeneous polynomial of order 2 Training and Testing Performance

Training: $\mathrm{R}=0.93171$

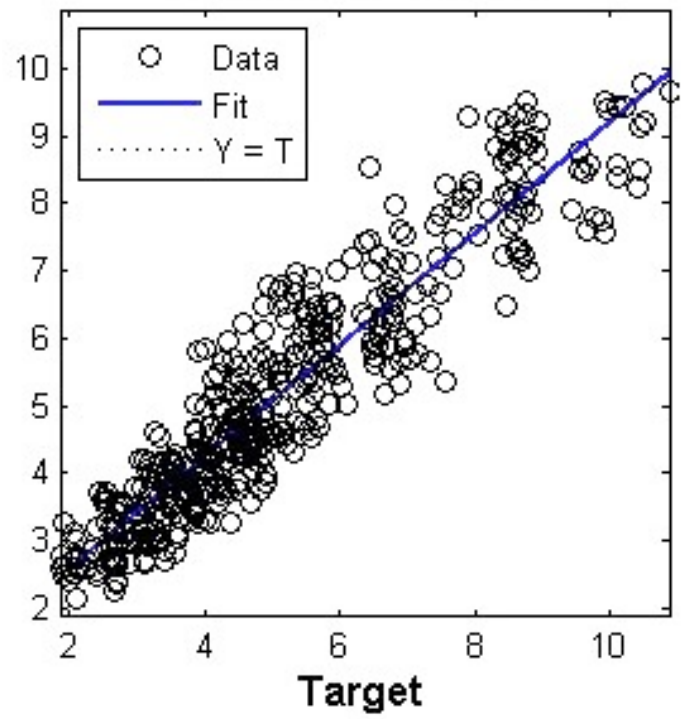

Test: $\mathrm{R}=0.93544$

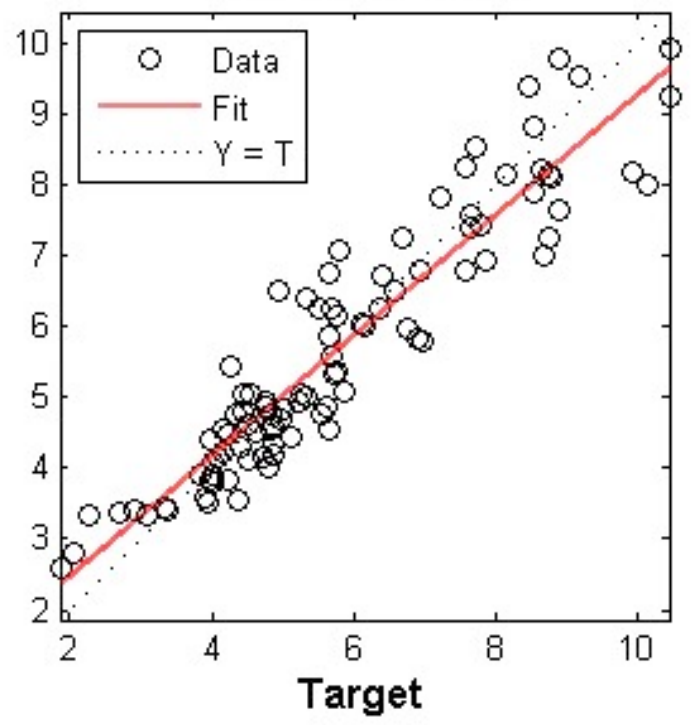

Fig. 2. Homogeneous polynomial of order 2 Training and Testing Performance 
Training: $\mathrm{R}=\mathbf{0} .96965$

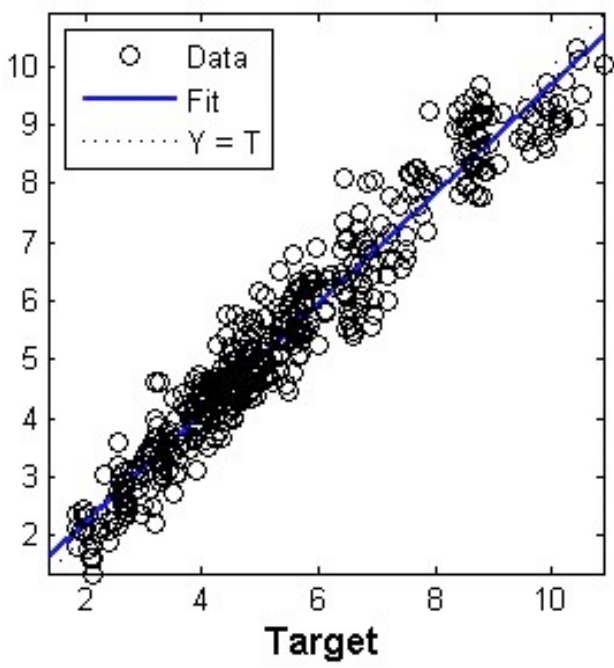

Test: $\mathrm{R}=0.94455$

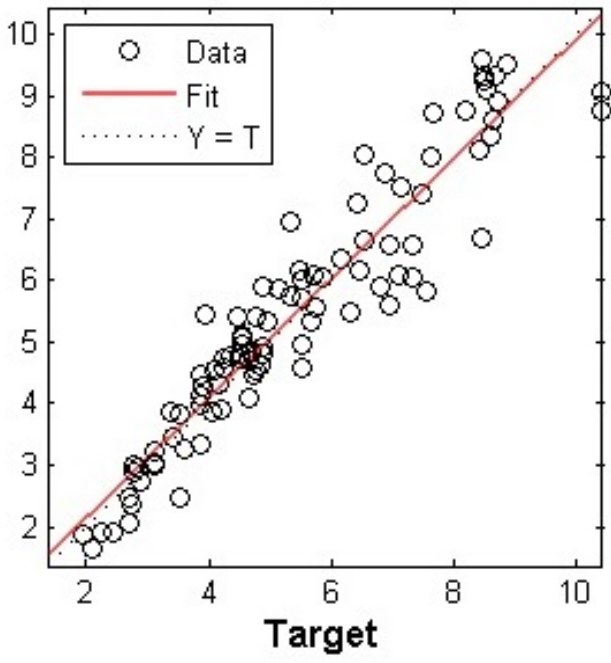

Fig. 3. Radial basis function Training and Testing Performance

Training: $\mathrm{R}=0.96101$

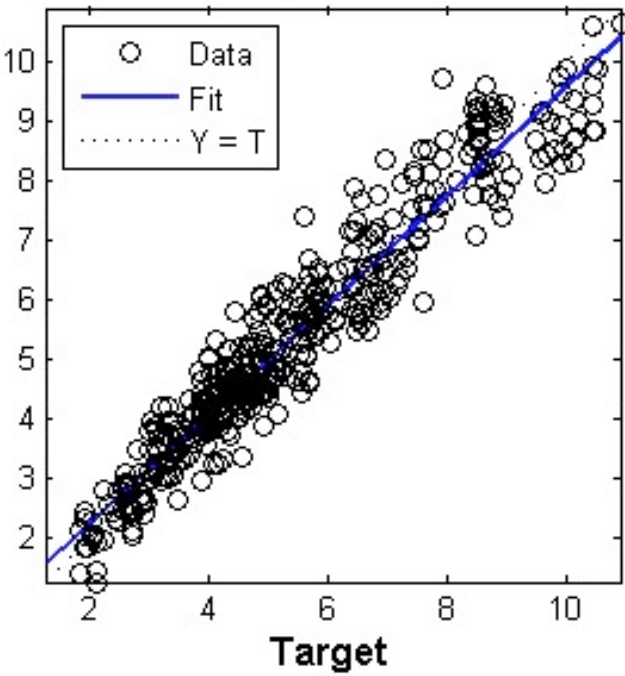

Test: $\mathrm{R}=0.95125$

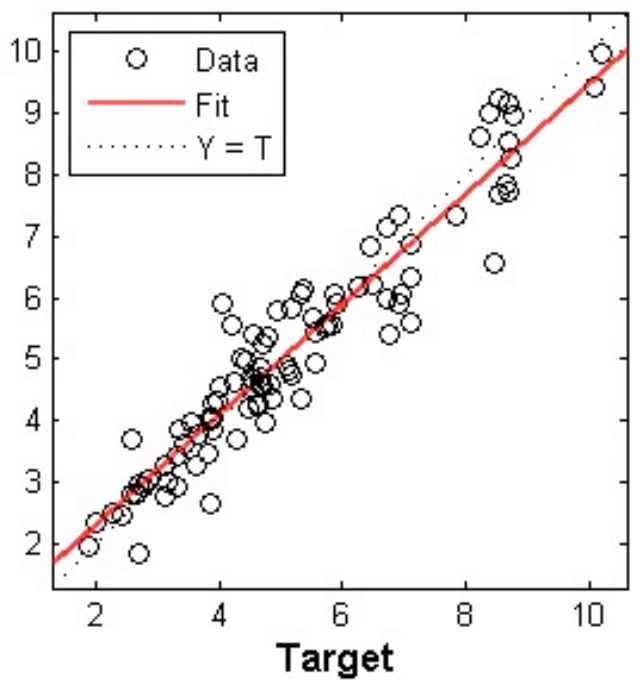

Training: $\mathrm{R}=0.96773$

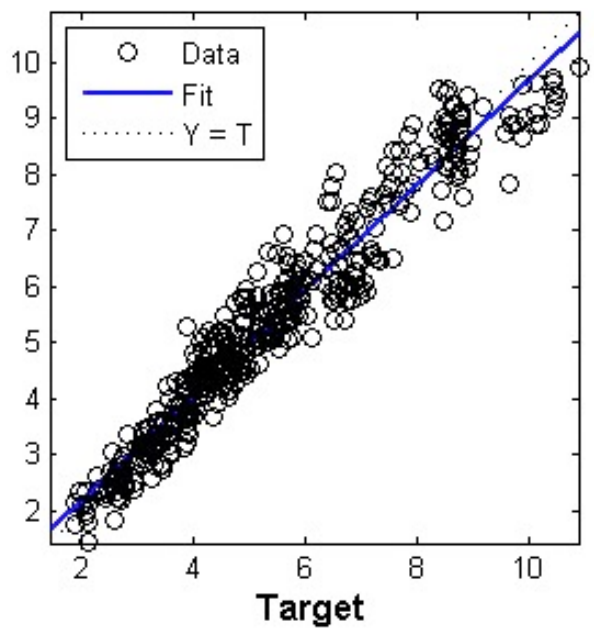

Test: $\mathrm{R}=0.94862$

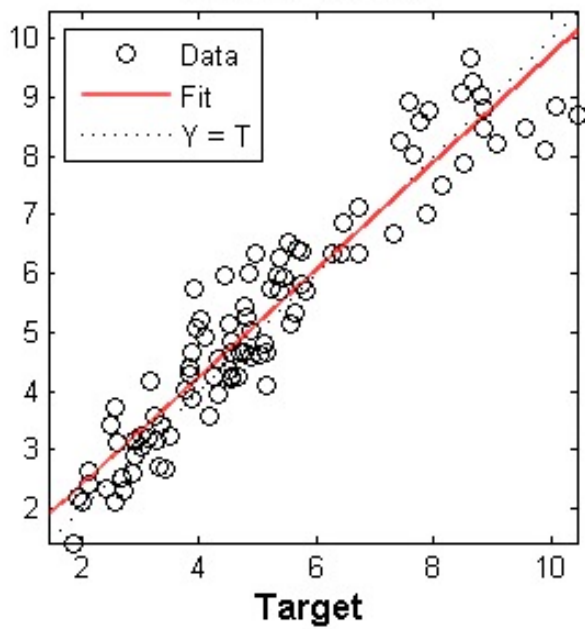

Fig. 5 sigmoid function Training and Testing Performance 


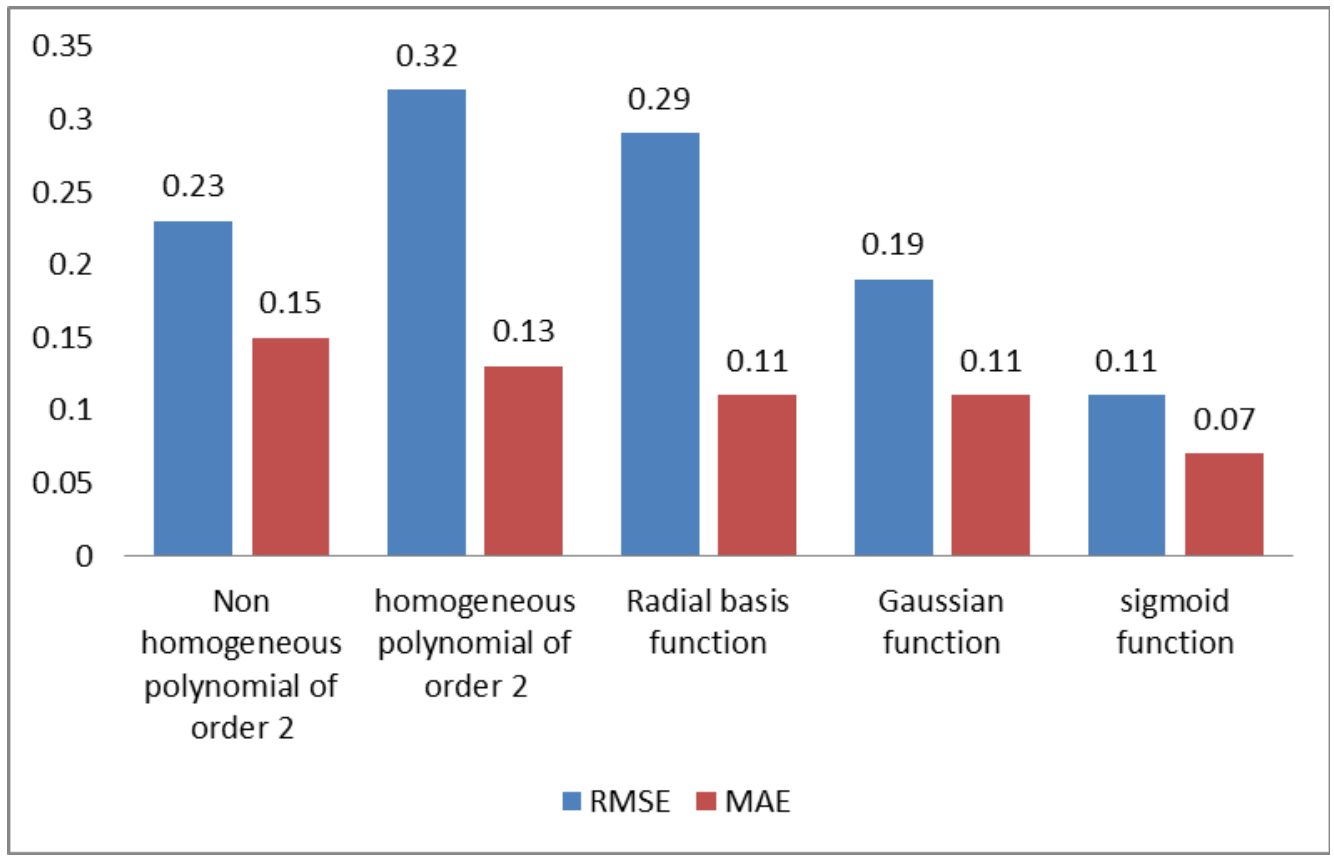

Fig. 6 RMSE and MAE of SVM models

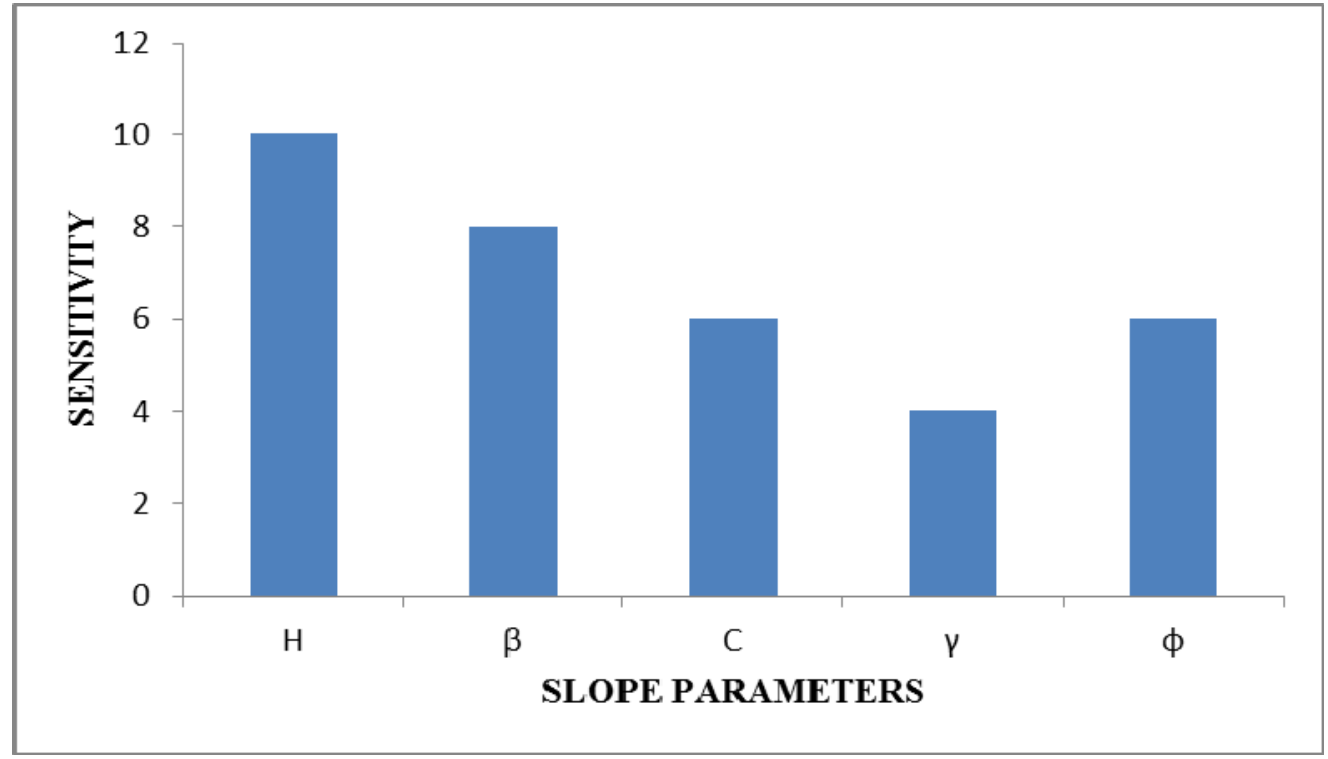

Fig. 7 Sensitivity Analysis

\section{VI.CONCLUSION}

The application of SVMs in stability analysis is studied and presented in this paper. The use of SVMs is very advantageous for the prediction of FoS because it can perform nonlinear regression efficiently for high dimensional data sets. As the SVM is mainly branded by the type of its kernel function, it is indispensable to elect the proper kernel for each precise application problem in order to promise acceptable results. The outcome of sigmoid function shows that SVMs have the capability to calculate the FoS with an adequate degree of exactness. The sensitivity analysis shows that the Height of slope and angle of slope are vital in this model.

\section{REFERENCES}

[1] Arora, K. R. Soil Mechanics and Foundation Engineering, $6^{\text {th }}$ ed Delhi. 2000.

[2] Atkinson, John. The mechanics of soils and foundations. $2^{\text {nd }}$ ed CRC Press, 2007.

[3] Bishop, Alan W. "The use of the slip circle in the stability analysis of slopes." The Essence of Geotechnical Engineering: 60 years of Géotechnique. Thomas Telford Publishing, 2008. 223-233.

[4] Cho, Sung Eun. "Probabilistic stability analyses of slopes using the ANN-based response surface." Computers and Geotechnics 36.5 (2009): 787-797. 
[5] Duncan, James Michael. "State of the art: limit equilibrium and finite-element analysis of slopes." Journal of Geotechnical engineering 122.7 (1996): 577-596.

[6] Fellenius, Wolmar. "Calculation of the stability of earth dams." Transactions of the 2nd congress on large dams, Washington, DC. Vol. 4. International Commission on Large Dams (ICOLD) Paris, 1936.

[7] Goh, Anthony TC, and S. H. Goh. "Support vector machines: their use in geotechnical engineering as illustrated using seismic liquefaction data." Computers and Geotechnics 34.5 (2007): 410-421.

[8] Kuo, Y. L., M. B. Jaksa, A. V. Lyamin, and W. S. Kaggwa. "ANN-based model for predicting the bearing capacity of strip footing on multi-layered cohesive soil." Computers and Geotechnics 36, no. 3 (2009): 503-516.

[9] Pal, Mahesh, and Surinder Deswal. "Modeling pile capacity using support vector machines and generalized regression neural network." Journal of geotechnical and geoenvironmental engineering 134.7 (2008): 1021-1024.

[10] Samui, P., and D. P. Kothari. "Utilization of a least square support vector machine (LSSVM) for slope stability analysis." Scientia Iranica 18.1 (2011): 53-58.

[11] Samui, Pijush. "Slope stability analysis: a support vector machine approach." Environmental Geology 56.2 (2008): 255.

[12] Samui, Pijush. "Support vector machine applied to settlement of shallow foundations on cohesionless soils." Computers and Geotechnics 35.3 (2008): 419-427.

[13] Shahin, Mohamed A., Mark B. Jaksa, and Holger R. Maier. "Artificial neural network applications in geotechnical engineering." Australian Geomechanics 36.1 (2001): 49-62.

[14] Spencer, E. "A method of analysis of the stability of embankments assuming parallel inter-slice forces." Geotechnique 17.1 (1967): $11-26$.

[15] Tinoco, Joaquim, A. Gomes Correia, and Paulo Cortez. "Support vector machines applied to uniaxial compressive strength prediction of jet grouting columns." Computers and Geotechnics 55 (2014): 132-140.

[16] Vapnik, Vladimir, Steven E. Golowich, and Alex Smola. "Support vector method for function approximation, regression estimation, and signal processing." Advances in neural information processing systems (1997): 281-287.

[17] Vinodhkumar S, Balaji S, "Artificial Neural Network Modelling and Economic Analysis of Black Cotton Soil Subgrade Stabilized with Flyash and Geotextile." International Journal of Earth Sciences and Engineering, Vol. 09, No. 01, pp. 81-86

[18] Zhao, Hong-bo. "Slope reliability analysis using a support vector machine." Computers and Geotechnics 35.3 (2008): $459-467$.

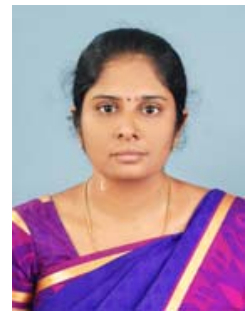

\section{AUTHOR PROFILE}

D. Ramya, completed B.E and M.E Computer Science and Engineering with first class, currently working as Assistant Professor in Department of Computer Science and Engineering, Sri Krishna College of Engineering and Technology, Coimbatore. Her area of research includes web technology, soft computing and its applications and data mining.

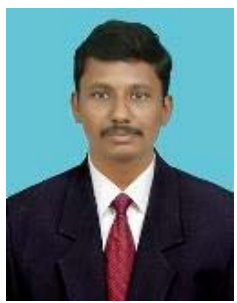

S.Vinodhkumar, completed B.E in Civil Engineering and M.E Geotechnical Engineering with first class, currently working as Assistant Professor in Department of Civil Engineering, Kongu Engineering College, Perundurai. His area of research includes Slope analysis, soft computing techniques and ground improvement. 